\title{
Desempeño de un sistema fotovoltaico autónomo frente a condiciones medioambientales de una región en particular
}

\author{
Edgar Darío Obando-Paredes*, Rubiel Vargas-Cañas
}

Departamento de Física, Facultad de Ciencias Naturales, Exactas y de la Educación, Universidad del Cauca, Popayán, Colombia

\begin{abstract}
Resumen
Este artículo presenta un método de modelado, simulación e implementación de un sistema fotovoltaico autónomo. El modelo está basado en un circuito equivalente del panel fotovoltaico; teniendo en cuenta la interacción de éste con los elementos que componen el sistema y con los parámetros medioambientales (radiación solar, velocidad del viento y temperatura ambiente) que influencian su funcionamiento. Este circuito equivalente esta descrito en términos de fotocorriente, un diodo, una resistencia en serie y una resistencia de juntura. La simulación del desempeño de este modelo se realiza teniendo en cuenta parámetros medioambientales recolectados en un periodo de un año y se valida comparando sus resultados con los de un modelo experimental. Los resultados obtenidos son comparables entre sí en términos de potencia.
\end{abstract}

Palabras Clave: panel fotovoltaico, radiación solar, Sistema Fotovoltaico, Modelo de sistema fotovoltaico.

Performance of an autonomous photovoltaic system due to environmental conditions of a particular region

\begin{abstract}
This paper presents a method of modelling, simulation and implementation of a stand-alone photovoltaic system. This model is based on the elements of an equivalent circuit of photovoltaic panel keeping in mind the interaction with elements of the system and environmental parameters (irradiation, temperature). The equivalent circuit is described in terms of photocurrent, a diode, a series resistor and a resistance shunt. The performance simulation this model are done taking into account environmental parameters collected over a period of one year and is validated with an experimental model which was built and comparable results were obtained in terms of power.
\end{abstract}

Key words: Photovoltaic panel, solar radiation, Stand-alone photovoltaic system, Matlab.

\section{Introducción}

El departamento del Cauca ocupa el primer lugar a nivel nacional en problemas de cobertura de energía eléctrica, representados en el número de viviendas sin electrificar; con un número aproximado de 47.955 viviendas sin servicio, le sigue La Guajira con 36.408, Córdoba con 34.173 y Antioquia con 30.599 (Colombia, Upme, 2014). Desde el punto de vista departamental, los municipios que presentan mayor número de viviendas que no pertenecen al Sistema Interconectado Nacional (SIN) son: Caldono con aproximadamente 4.214, El Tambo con 4.156, La Vega con 1.114, Bolívar con 2.882, mientras que en Popayán, su capital hay 114 viviendas sin el servicio de electricidad. Es en este punto donde el uso de fuentes no convencionales de energía renovables (FNCER) surge como una alternativa de conexión y generación de electricidad, planteando una nueva forma en el manejo, masificación e implementación de sistemas energéticos. Este es el caso de la energía solar fotovoltaica, donde se aprovecha directamente la radiación incidente del Sol para generar electricidad. Se prevé que con el estudio y apropiación de las técnicas de implementación de sistemas basados en energía solar y en fuentes renovables de energía, éstas podrían proveer las tres quintas partes de mercado de la electricidad del mundo disminuyendo en dos quintas partes el mercado de los combustibles y reduciendo de la misma manera la huella de carbono (IRENA, 2011).

La energía solar fotovoltaica, ha sido ampliamente estudiada, países como Canadá, Brasil, Algeria, España, Alemania, India y Japón (Verma S, Verma H. K., Md. Mohiddin K, 2011; Semaoui S, Hadj Arab A, Bacha S. Azoui B, 2013; Krismadinata, N. Rahima H, Jeyraj Selvaraja, 2013; Cibira G, Košĉová M, 2014; Salmi T, Bouzguenda M, Gastli A, Masmoudi, 2012; Qi Chen, Ming Zhu, 2012; I.H. Altas, and A.M. Sharaf, 2007), quienes han sido pioneros en el estudio de implementación de sistemas fotovoltaicos para producción de energía eléctrica. En estos estudios se ha llegado a modelos de desempeño entre los que cabe destacar:

\footnotetext{
*Correspondencia:

Edgar Darío Obando-Paredes, dariobando@unicauca.edu.co

Recibido: 12 de noviembre de 2015

Aceptado: 23 de noviembre de 2015
} 
el modelo de desempeño Duffie \& Beckman (Duffie J, Beckman W.A, 1980), modelo de desempeño King (D.L. King, W.E. Boyson, J.A. Kratochvill, 2004) y Modelo de desempeño Masters (Masters G, 2004), los que demuestran la relación directa entre variables medioambientales (radiación solar, temperatura ambiente, velocidad del viento) y la producción de electricidad en términos de índice de desempeño. En Colombia el grupo de Materiales Semiconductores y Energía Solar de la Universidad Nacional de Colombia ha estudiado e implementado sistemas fotovoltaicos midiendo la potencia entregada por estos en diferentes regiones del país, estudiando el desempeño en términos de electricidad en dichas regiones teniendo en cuenta variables propias, permitiendo y abriendo camino para la investigación aplicada en la validación de modelos físicos que representen el comportamiento real del panel fotovoltaico ante condiciones medioambientales específicas (Gordillo G \& L Angulo, 1992). En contraparte, en este trabajo se muestra el desempeño de un sistema fotovoltaico teniendo en cuenta las variables medioambientales que definen una región (radiación solar, velocidad del viento y temperatura ambiente), en trabajos anteriores no se había considerado en el desempeño final el efecto de considerar la velocidad del viento. Hasta la fecha de revisión bibliográfica no se encontró estudio del comportamiento energético de sistemas fotovoltaicos en términos de potencia entregada en la ciudad de Popayán ni en el departamento del Cauca.

Este trabajo muestra un estudio de la generación de potencia eléctrica en base a un modelo matemático que describe las propiedades eléctricas (corriente, voltaje, resistencia) de un sistema fotovoltaico, dependiendo directamente de variables medio ambientales (radiación solar, temperatura ambiente y velocidad del viento) propias del municipio de Popayán. Este estudio se llevó a cabo en dos etapas: en la primera etapa se midieron, continuamente, la variables medioambientales y se simularon los resultados de potencia entregada por un sistema fotovoltaico basado en un modelo matemático; en la segunda etapa se validaron los resultados de la simulación implementando un prototipo experimental con miras a ajustar el modelo teórico.

El resto de este documento se organiza de la siguiente manera: en primer lugar, se realiza una breve revisión de los sistemas fotovoltaicos, clasificándolos teniendo en cuenta la etapa de almacenamiento, la región de implementación del sistema, las variables medioambientales que se adquirieron y los diferentes modelos matemáticos que se utilizan para evaluar el desempeño de un arreglo fotovoltaico en términos de potencia. Luego se presenta una descripción del diseño del prototipo instalado y su funcionamiento, la simulación del modelo teórico y su comparación con los resultados arrojados por el prototipo. Finalmente se realiza ajustes al modelo teórico, con el fin de minimizar el error obtenido y hacerlo más cercano a la realidad, al tener en cuenta variables medioambientales tales como la velocidad del viento la cual no es de uso común en los modelos matemáticos tradicionales.

\section{Metodología}

Con el fin de plantear un modelo matemático que pueda predecir el comportamiento energético de un sistema fotovoltaico a partir de las condiciones medioambientales específicas de un lugar se realizó un circuito equivalente en términos de variables eléctricas, teniendo en cuenta el modelado de un diodo. Paralelamente, se llevó a cabo la medición de las variables medioambientales como temperatura, radiación, y velocidad del viento. Posteriormente se simula el desempeño arrojado por el modelo matemático, después de realizada la simulación, se implementó un prototipo para validar los resultados obtenidos en la simulación y así ajustar el modelo al comportamiento real.

\section{Región de implementación}

El lugar de estudio fue la ciudad de Popayán, ubicada en el centro del departamento del Cauca (Suroccidente colombiano, 2० 26' 49” N, 76 35' 49”' W, 1750 m.s.n.m). Popayán es una ciudad histórica por excelencia con un clima templado y temperatura promedio de $20^{\circ} \mathrm{C}$, condiciones meteorológicas variables entre clima seco y lluvias. La radiación solar en la zona oscila entre 4.0 y $4.5 \mathrm{Kwh} / \mathrm{m}^{2}$ promedio multianual, como lo muestra la Figura 1S, http://www.raccefyn.co/index.php/raccefyn/article/ downloadSuppFile/301/1268.

\section{Tipos de sistemas fotovoltaicos}

El fundamento de esta metodología se consignó en la información suplementaria 1, http://www.raccefyn.co/index. php/raccefyn/article/downloadSuppFile/301/1271.

\section{Modelos de desempeño}

El fundamento de esta metodología se consignó en la información suplementaria 2, http://www.raccefyn.co/index. php/raccefyn/article/downloadSuppFile/301/1272. Figura 2S, http://www.raccefyn.co/index.php/raccefyn/article/ downloadSuppFile/301/1269.

\section{Implementación del sistema fotovoltaico autónomo}

Para el estudio de la potencia entregada por un sistema fotovoltaico bajo las características medioambientales propias de la ciudad de Popayán, se implementó un prototipo de sistema fotovoltaico autónomo. Teniendo contacto directo con variables medioambientales tales como temperatura ambiente, velocidad del viento y radiación incidente. Un diagrama de bloques del prototipo de sistema autónomo se muestra en la Figura 3S, http://www.raccefyn.co/index.php/ raccefyn/article/downloadSuppFile/301/1270.

El fundamento metodológico de componentes y funcionamiento del sistema de medición se consignó en la información suplementaria 3S, http://www.raccefyn.co/ index.php/raccefyn/article/downloadSuppFile/301/1273. 


\section{Resultados}

\section{Adquisición de datos meteorológicos}

Las variables medioambientales (temperatura ambiente, radiación solar, velocidad del viento), fueron censadas durante un año en la estación meteorológica ubicada en la Facultad de Ingeniería Civil de la Universidad del Cauca. De este periodo un $70 \%$ de los datos fueron usados para la simulación de potencia, y un 30\% correspondiente al periodo de instalación del prototipo y su posterior evaluación de rendimiento energético. Las variables ambientales sensadas, promedio diario y mensual se muestran en las Figuras $1 \mathrm{y}$ 2 respectivamente.

\section{Resultados entregados por el prototipo}

Para la medición de potencia se implementa el prototipo en el mismo lugar donde está ubicada la estación meteorológica. Los datos se obtuvieron mediante la medición indirecta de potencia, calculada mediante la resistencia de carga implementada y el voltaje que resulta cada media hora. Un periodo de muestreo diario desde las 8:00 hasta las 19:00 (GMT-5:00). Los resultados para potencia medida y potencia simulada (teórica) en el intervalo diario de muestreo, se muestran en la Figura 3; comparando los resultados se encuentra un error cuadrático medio del $17 \%$, un valor bajo teniendo en cuenta que el sistema se sobredimensiona en su implementación y posterior cálculo de potencia total, para prevenir perdidas inherentes al sistema producidas debido al cableado, dispositivos, estado de carga e intensidad de descarga de la batería.

En la Figura 4, se muestran los resultados de potencia medida y potencia simulada, en el intervalo horario de muestreo, en este caso el error cuadrático medio es del
$1,8 \%$, considerablemente menor que en el caso diario, debido principalmente a la tasa de toma de datos entre muestras. En general, para minimizar el error cuadrático medio existente, el prototipo debe censar datos en el mismo rango y tasa que la estación meteorológica.

Teniendo en cuenta las Figuras 3-4 y el modelo matemático de la Ecuación (6S), en el cálculo de la potencia entregada por un arreglo fotovoltaico se deben tener en cuenta características como: el número de celdas interconectadas, la temperatura en la celda y corriente de saturación; parámetros de manufactura y propios de cada técnica de producción de paneles fotovoltaicos. En la aplicación de este modelo, no se tienen en cuenta variables medioambientales tales como: velocidad del viento, pluviosidad en la región, entre otros. Debido al efecto Joule presente en los cables funcionando en corriente directa se deben tener en cuenta pérdidas energéticas existentes al interconectar los dispositivos, además del estado de carga y la intensidad de descarga de la batería; para minimizar estas pérdidas se hace necesario sobredimensionar el sistema (M.S. Mahmodian, R. Rahmani, E.Taslimi, S. Mekhilef, 2012).

\section{Ajuste del modelo utilizado, teniendo en cuenta la velocidad del viento}

Al realizar la instalación de sistemas fotovoltaicos en regiones donde existe un alto índice de radiación solar, está directamente relacionado con la velocidad del viento en esas regiones puesto que la acción de rayos solares, hace que masas de aire se transporten de un lugar a otro debido a un gradiente de temperatura. Es así como se realizaron ajustes al modelo utilizado, integrando la Ecuación (8S) al modelo eléctrico inicial, se llega a la expresión que
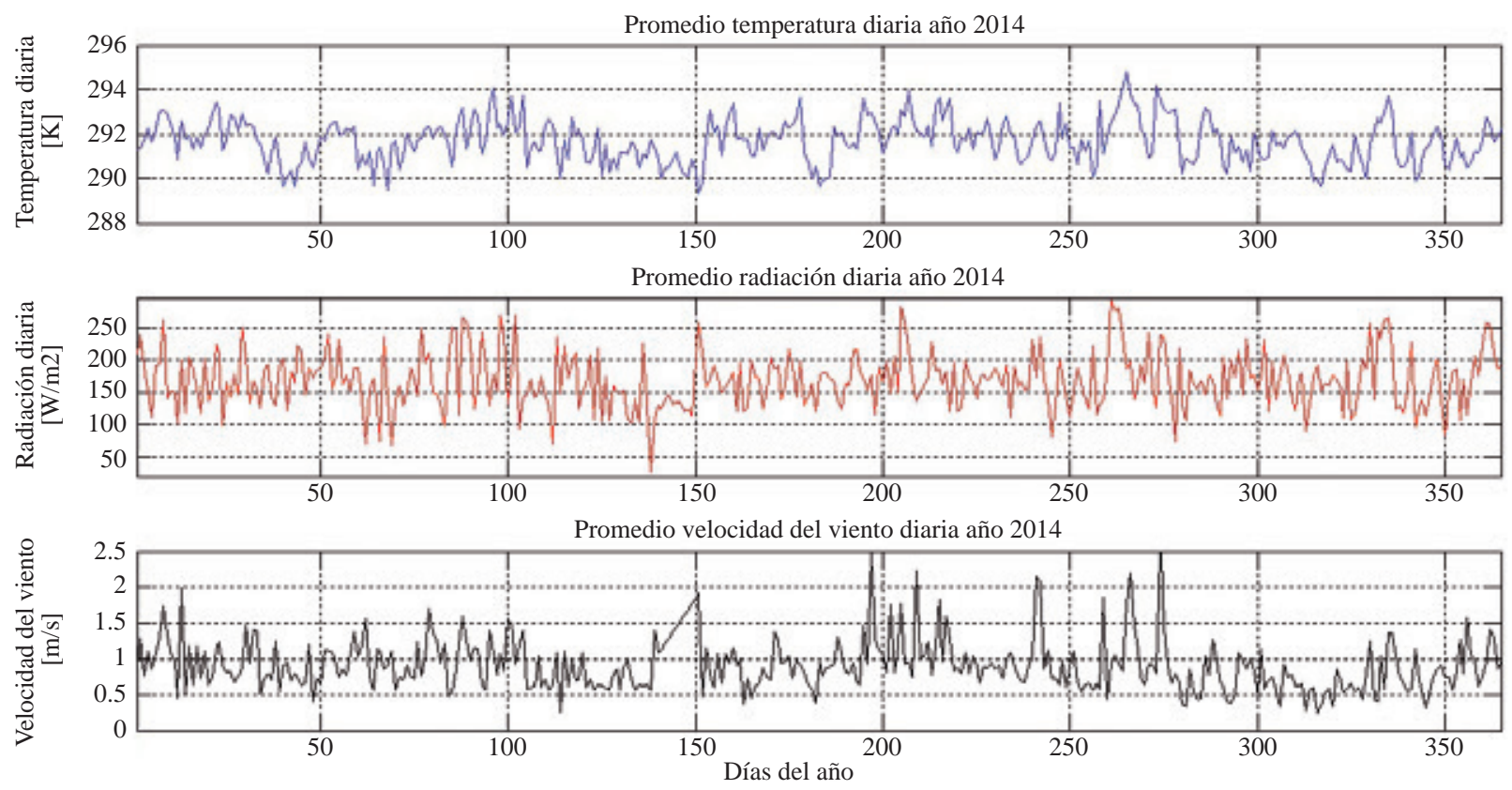

Figura 1. Variables medioambientales promedio diario año 2014 en la ciudad de Popayán. 

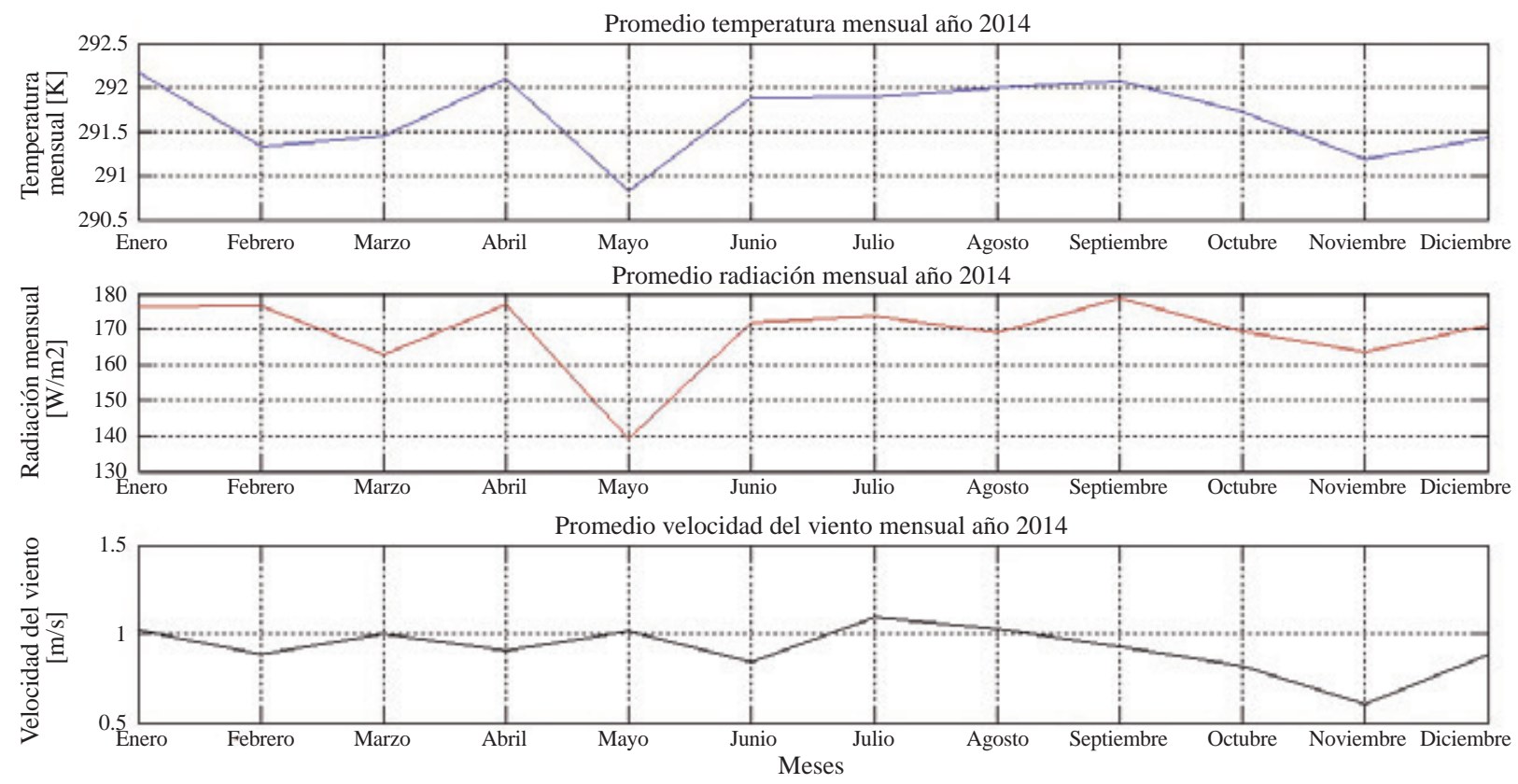

Figura 2. Variables medioambientales promedio mensual año 2014 en la ciudad de Popayán.

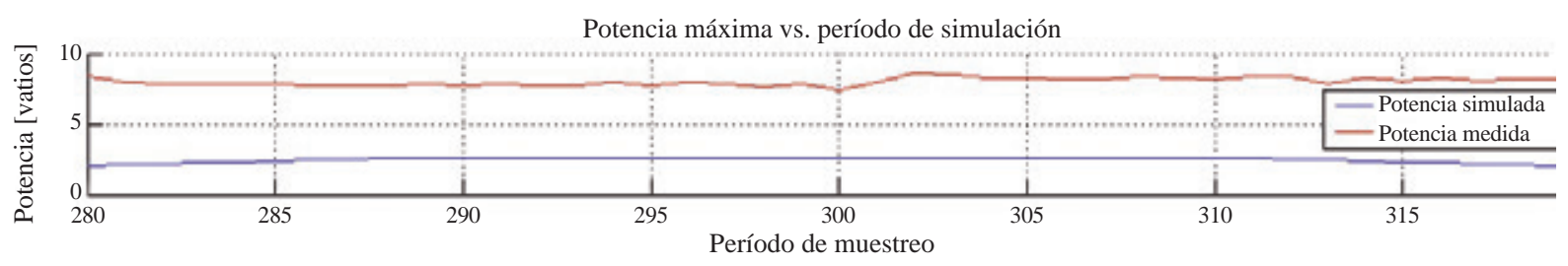

Figura 3. Potencia simulada vs Potencia medida para el periodo de muestreo.

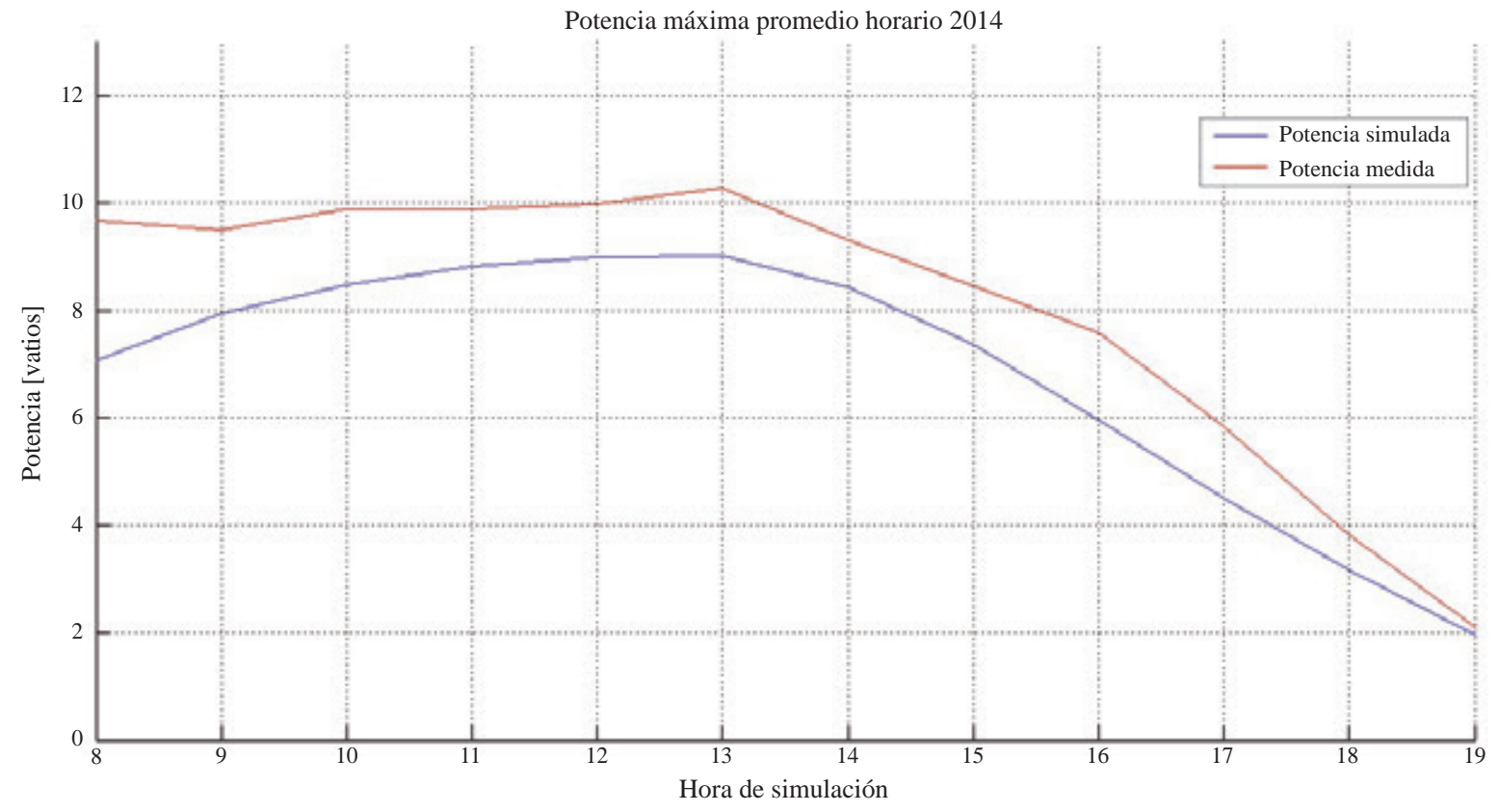

Figura 4. Potencia simulada vs Potencia medida por horas de muestreo. 
relaciona las tres variables medioambientales: radiación solar $G$, velocidad del viento $V$ y temperatura $T$, representado en la Ecuación 1, en aras de lograr un mejor desempeño en términos de potencia entregada por hora, presentado en la Figura 5. Este ajuste muestra aumento en el error cuadrático medio, pasando de $1.8 \%$ a $1.9 \%$, el aumento de este error, se debe a la asignación de los coeficientes $a$ y $b$ en la Ecuación final, parámetros dados por el fabricante y que dependen exclusivamente de las condiciones en que se hayan efectuado las medidas (M.S. Mahmodian, et al., 2012). En cambio la potencia entregada final aumenta aproximadamente un $30 \%$ al integrar la velocidad del viento en el modelo utilizado, pasando en promedio de entregar 8.25 Vatios a entregar 11.04 vatios promedio máximo.

$$
\begin{aligned}
P=V & {\left[N_{p} *\left(\left(\left(I_{s c r}+\mathrm{K}_{i} *\left(T_{c}-\mathrm{T}_{r e f}\right)\right) * \frac{\mathrm{G}}{1000}\right)\right)-I_{r r} *\right.} \\
& \left.\left.\left(\mathrm{e}\left(\frac{q * V}{N_{S} * k *\left(\left[G_{T} *\left\{e^{a+b * V}\right\}+\mathrm{T}_{a}\right]+\frac{G_{T}}{G_{r e f}} \Delta T\right) * A}\right)-1\right)\right)\right]
\end{aligned}
$$

Los resultados de desempeño simulado y medido por días, presenta un comportamiento similar, teniendo un aumento de cerca del $37 \%$ si se considera la velocidad del viento; pasando de 3.97 vatios a 7.64 vatios en promedio máximo. La Figura 6, muestra la potencia teniendo en cuenta la velocidad del viento y las variables medioambientales consideradas anteriormente. En este punto cabe resaltar que el error cuadrático medio disminuyo a un 3,9\%, que de acuerdo al modelo de desempeño King, et al., es aceptable, puesto que usando este modelo se presenta una diferencia de exactitud en un intervalo de entre 2,4\% y 5,4\% (D.L. King, et al., 2004).

En las Figuras 7 y 8 se muestran la comparación de desempeño entre los modelos teóricos existentes para el periodo de muestreo, tanto horario como diario. De estas graficas se infiere que el desempeño aumenta al tener en cuenta la velocidad del viento en el modelo puesto que de acuerdo con la Ecuación (8S), existe una relación directa entre la temperatura de la celda, $T_{C}$, dependiente del viento y la radiación incidente. Hay que tener en cuenta la velocidad de recombinación electro-hueco que depende directamente de la temperatura a la que se encuentra el sustrato.

\section{Conclusiones}

En este artículo, se desarrolló un método para determinar la potencia entregada por un sistema fotovoltaico autónomo, instalado en la ciudad de Popayán. El modelo recomendado a utilizar para el cálculo de potencia, depende directamente de factores medioambientales y características propias de la manufactura y técnica de producción de los módulos fotovoltaicos. Este modelo fue simulado y validado experimentalmente, implementando un prototipo de sistema fotovoltaico autónomo. Como resultado de esta implementación y simulación se compararon los valores de potencia arrojados por el prototipo con los valores de potencia simulados, encontrando una relación existente entre algunas variables medioambientales y eléctricas que no se consideran en el modelo matemático, como la velocidad del viento, pero considerada indirectamente en la medida de potencia final por el prototipo. Para el cálculo de potencia se reajustó el modelo teniendo en cuenta la velocidad del viento,

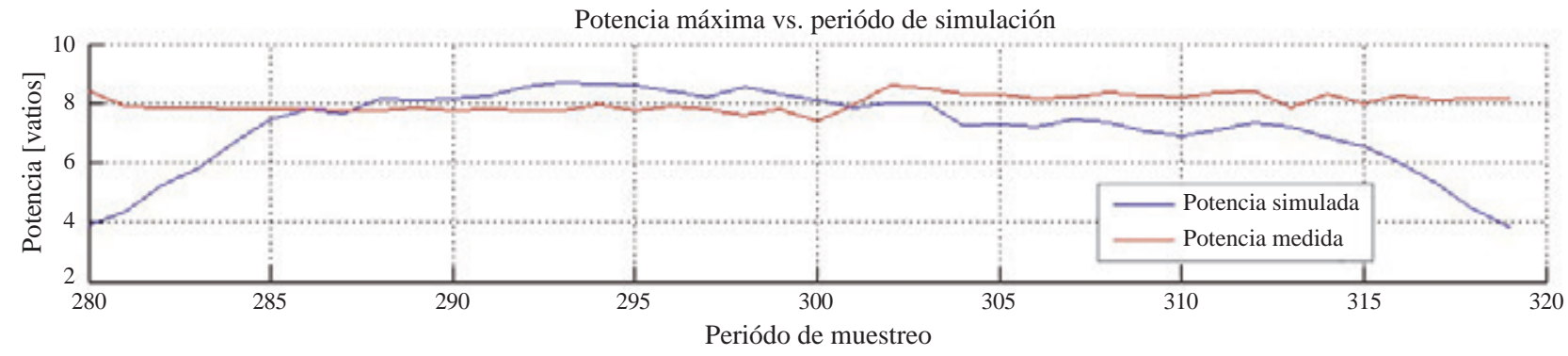

Figura 5. Potencia simulada vs Potencia medida por horas de muestreo, teniendo en cuenta la velocidad del viento.

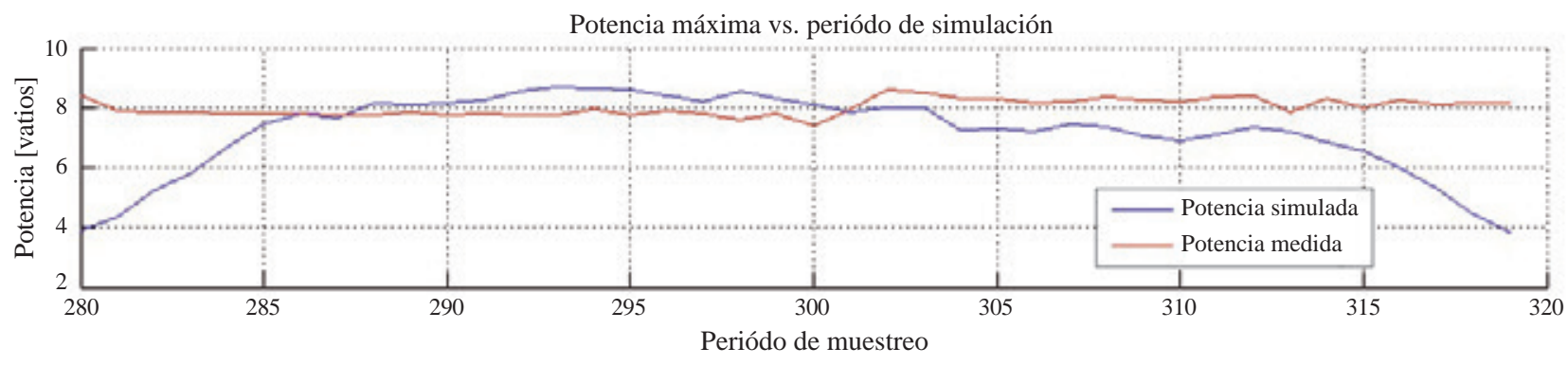

Figura 6. Potencia simulada vs Potencia medida para el periodo de muestreo, teniendo en cuenta la velocidad del viento. 


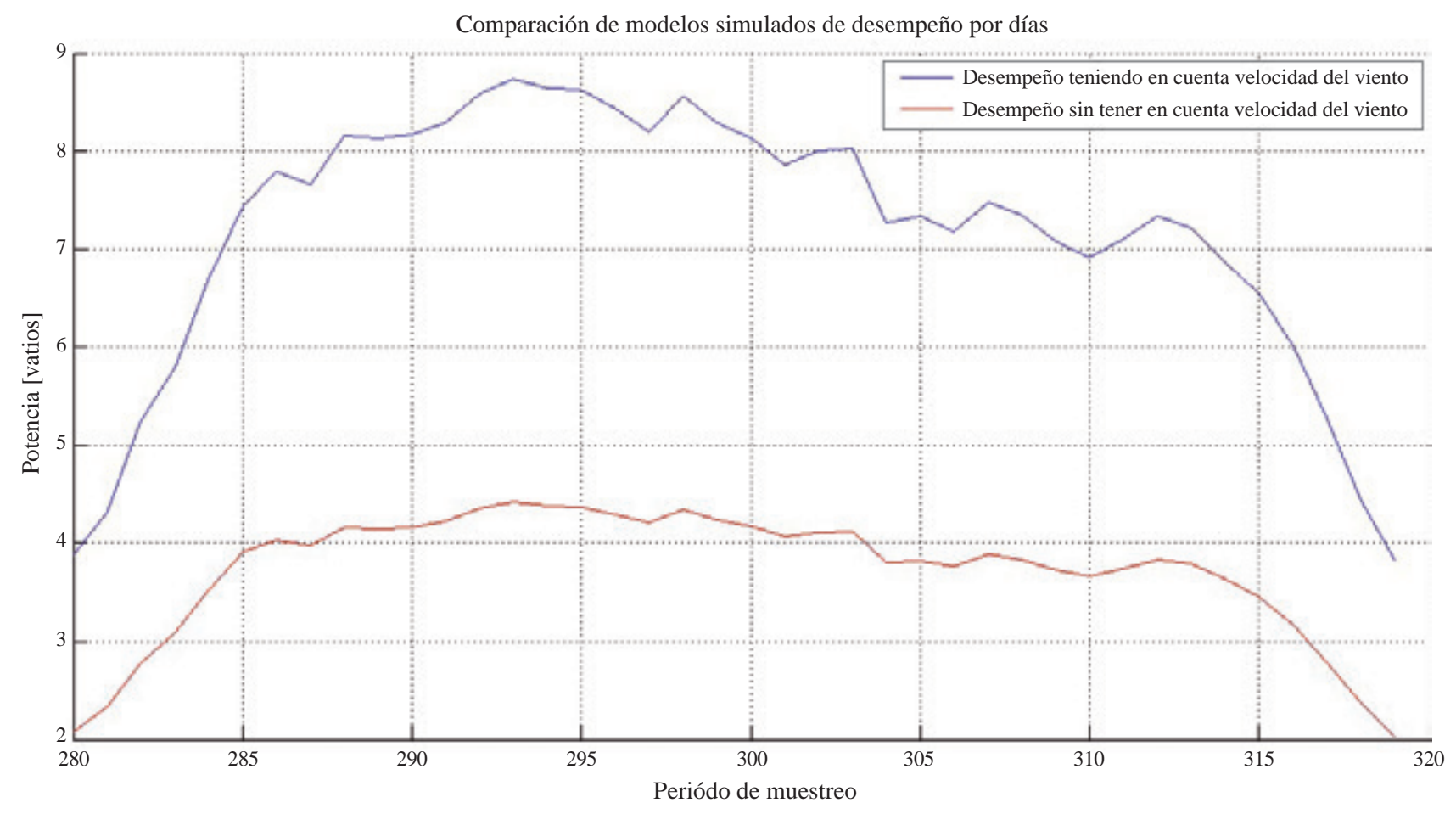

Figura 7. Comparación de desempeño al tener en cuenta velocidad del viento, en el periodo de muestreo.

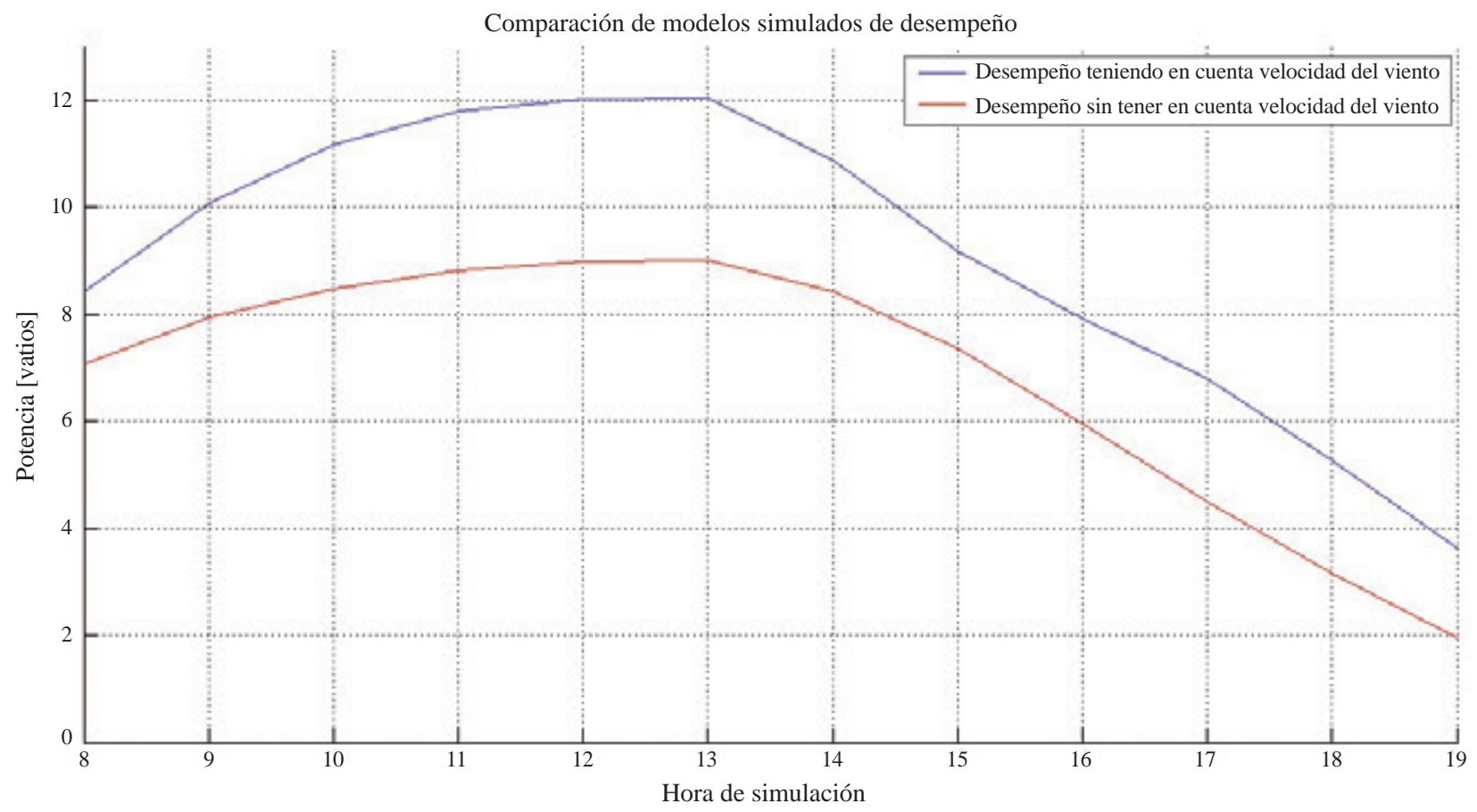

Figura 8. Comparación de desempeño al tener en cuenta velocidad del viento, por horas de muestreo.

puesto que trabajos anteriores solo consideran un modelo matemático dependiente de la temperatura y la radiación; en este trabajo se integra las tres variables medioambientales necesarias para definir una región geográfica en particular.
Para estudios posteriores se recomienda el modelado de fuentes no renovables de energía a gran escala debido a que proporciona herramientas para un óptimo diseño y procesos eficientes de gestión energética. 


\section{Información suplementaria}

Información suplementaria 1: Tipos de sistemas fotovoltaicos.

Información suplementaria 2: Modelos de desempeño.

Información suplementaria 3: Componentes del prototipo implementado.
Figura 1S. Mapa radiación solar departamento del Cauca (Colombia, UPME, 2005).

Figura 2S. Circuito Equivalente celda solar

Figura 3S. Prototipo sistema fotovoltaico autónomo.

\section{Agradecimientos}

Los autores desean expresar su agradecimiento al Ingeniero Luis Jorge Gonzales por su infinita colaboración en el préstamo de las instalaciones de la estación meteorológica de la Facultad de Ingeniería Civil de la Universidad del Cauca, al señor Tirso Mazabuel, Jefe del laboratorio de Electrónica de la Facultad de Ingeniería Electrónica y Telecomunicaciones de la Universidad del Cauca, al Ingeniero Ricardo Muñoz y al Ingeniero Carlos Felipe Rengifo docente de la Facultad de Ingeniería Electrónica y Telecomunicaciones de la Universidad del Cauca.

\section{Conflicto de intereses}

Los autores declaran no tener ningún conflicto de intereses.

\section{Bibliografía}

Cibira G \& Košĉová M. (2014). Photovoltaic module parameters acquisition model. Applied Surface Science. 312: 74-80.

Colombia. Ministerio de Minas y energía. Ley 1715 de 2014 sobre integración de Fuentes no convencionales de energía renovable al sistema interconectado nacional.

Colombia. Unidad de Planeación Minero Energética -UPME. (2005). Atlas Radiación Solar. http://www.upme.gov.co/ Docs/Atlas_Radiacion_Solar/1-Atlas_Radiacion_Solar.pdf.

Colombia. Unidad de Planeación Minero Energética UPME. (2014). Cobertura Energética Versión preliminar a 2014 en Colombia. www.upme.gov.co/Docs/PIEC/PIEC_UPME_ 2010_2014_Prelimar.pdf.

Colombia. Unidad de Planeación minero energética-UPME. (2014). Integración de las energías renovables no convencionales en Colombia, CONVENIO ATN/FM-12825-CO. La Imprenta Editores S.A. 2015 P:99-101.

Duffie J, Beckman W.A. (1980). Solar Engineering of Thermal Processes. Wisconsin. Jhon Willey \& Sons.

Gordillo G \& L Angulo. (1992). Desarrollo de un sistema de monitoreo de módulos fotovoltaicos. (Tesis de pregrado). Universidad Nacional de Colombia. Bogotá.

I.H. Altas, \& A.M. Sharaf. (2007). A photovoltaic array simulation model for Matlab-Simulink GUI environment, International Conference on Clean Electrical Power ICCEP’07, Capri, pp. 341-345.
IRENA (2011). Opportunities for Economic Value Creation along the Solar and Wind Value Chain, Multilateral Working Group on Solar and Wind Energy Technologies, MWG Solar \& Wind.

Jäger K, Isabella O, H.M. Smets A, Van Swaaij R \& Zeman M. (2014). Solar Energy Fundamentals, Technology, and Systems. (p.p.28-35) Delft: Delft University of Technology.

Kalogirou, S. (2009). Solar energy engineering: processes and systems. (3 ed.). United Kingdom: Elsevier Book AID International.

King D.L, Boyson W.E \& Kratochvill J.A. (2004) Photovoltaic Array Performance Model, Sandia report SAND2004-3535.

Krismadinata, N. Rahima H \& Jeyraj Selvaraja. (2013). Photovoltaic module modeling using simulink/matlab. Procedia Environmental Sciences (17): 537-546.

Labouret, Villoz M. (2009). Solar Photovoltaic Energy. The Institution of Engineering and Technology of United Kingdom.

Mahmodian MS, Rahmani R, Taslimi E, Mekhilef S. 2012. Step By Step Analyzing, Modeling and Simulation of Single and Double Array PV system in Different Environmental Variability. International Conference on Future Environment and Energy IPCBEE 28: 37-42.

Masters G. Renewable and Efficient Electric Power Systems 2004 (4. Ed). New York: John Wiley \& Sons, Inc.

Qi Chen, Ming Zhu. (2012). Photovoltaic Module Simulink Model for a Stand-alone PV System. Physics Procedia (24): 94-100.

Salmi T, Bouzguenda M, Gastli A \& Masmoudi A. (2012). MATLAB/Simulink Based Modelling of Solar Photovoltaic Cell. International Journal of Renewable Energy Research, 2 (2): 213-218.

Semaoui S, Hadj Arab A, Bacha S \& Azoui B. (2013). Optimal Sizing of a Standalone photovoltaic system with energy management in isolated areas. Elsevier-Energy Procedia 36: 358-368.

Verma S, Verma H. K \& Md. Mohiddin K. (2011). Modeling $\&$ analysis of standalone photovoltaic System. Ijret: International Journal of Research in Engineering and Technology. 02 (11): 259-265. 